\title{
Enhanced Delivery of Chemotherapeutic Alkylating Agents into Prostate Cancer Cells Employing the Androgen Receptor as Delivery Vehicle
}

\author{
Marvin Rubenstein ${ }^{1,2,3 *}$, Courtney MP Hollowell ${ }^{2}$ and Patrick Guinan ${ }^{1,2,4}$ \\ ${ }^{1}$ Division of Cellular Biology, Hektoen Institute for Medical Research, 2240 W. Ogden Ave., Chicago, IL 60612, United States \\ ${ }^{2}$ Division of Urology, Stroger Hospital of Cook County, Chicago, IL 60612, United States. \\ ${ }^{3}$ Departments of Biochemistry and Urology, Rush University Medical Center, Chicago, IL 60612, United States \\ ${ }^{4}$ Departments of Urology, University of Illinois at Chicago, and Rush University Medical Center, Chicago, IL, 60612, United States
}

\begin{abstract}
Effective chemotherapy for tumors, particularly those of disseminated prostate cancer, is limited by the inability of effective agents to distinguish between tumor and non-tumor cells, their inability to be delivered in sufficient dosage to susceptible targets (within tumor cells and organelles) and the development of tumor resistance. Overall chemotherapy for prostate cancer is relatively ineffective, providing only months of additional survival time for recurrent disease. While most recurrent and advanced stage tumors are resistant to chemotherapy, there may be a window of opportunity when new types of innovative therapy can be employed. Until proven, this approach could be employed either as adjuvant treatment for localized disease primarily treated by surgery or radiation; or for advanced cases, in combination with traditional chemotherapy protocols which currently provide only a limited improvement in survival.

The innovative therapy being proposed here employs the tumors' endogenous androgen receptor (AR) not only as a tumor specific marker for identification and specificity, but also as a delivery vehicle. By coupling the DNA cross linking chemotherapeutic cyclophosphamide (Cytoxan) to an androgen receptor ligand (AR agonist; R1881) cytotoxic chemotherapy can be delivered not only specifically to those cells which express AR but also, through the natural intracellular transit of this activated receptor, specifically direct the drug into the nucleus and transcriptionally active DNA sites. When localized, opposing DNA strands would be cross linked preventing DNA transcription and replication, leading to cell death. Normal androgen activation (provided by the R1881 agonist) mediated via the bound receptor would make the cell even more susceptible to Cytoxan action. An in vitro assay to determine any enhanced activity of Cytoxan combined with R1881 vs Cytoxan alone is easily performed and described.
\end{abstract}

Keywords: Prostate cancer; Delivery; Antisense; Chemotherapy

\section{Introduction and Background}

The effectiveness of chemotherapy has always been compromised by the side effects related to the distribution of highly toxic agents throughout the body and their effects on normal cells. This is particularly acute for those agents which are either not specifically targeted or whose method of toxicity is not selective for tumor cells. Therefore, the development of specific targeting strategies for effective agents has been an area of intense interest. Improvements in delivery and selectivity should be based on: A) The intended target's distribution and stability; B) The molecular configuration of agent; and C) The targeting strategy and delivery vehicle. For prostate cancer these considerations specifically include the following:

\section{Target distribution and stability}

Although early stage prostate cancer is treatable by surgical resection, radiation and hormone deprivation, it tends to recur and metastasize. Early and well differentiated tumors are androgen sensitive and their activity is regulated through the activity of the AR acting as a DNA binding transcription factor [1]. Among the genes transcriptionally regulated are those encoding prostate specific antigens: Prostate specific antigen (PSA) [2] and prostate specific membrane antigen (PSMA) [3].

Androgen receptor (AR): Well differentiated prostate tumors express the AR. Also known as NR3C4 (nuclear receptor subfamily 3 , group C, member 4), this nuclear receptor is cytoplasmically expressed and binds to either testosterone or its dihydrotestosterone (DHT) metabolite. The activated AR/ligand complex is then transported into the nucleus where it specifically binds to active DNA regions and serves as a transcription factor for prostate specific gene expression.
PSA and PSMA: Prostate specific antigen (PSA) is a secreted kallikrien related peptidase marker associated with prostate cancer recurrence and progression. It's often employed as a screening tool and has been evaluated as a target for directed therapy. In contrast, prostate specific membrane antigen (PSMA) is a transmembrane bound receptor with folate hydrolase activity. It is endocytic and negatively regulated by androgen [3] making it useful as both a delivery agent and marker for tumor imaging. PSA and PSMA are considered targets for an activated immune system and have been included in prostate cancer vaccines, such as Provenge developed by Dendrion employing PSA and ALPHAVAX ARV developed by the consortium of Cytogen and Progenics Pharmaceuticals.

\section{Molecular Agents}

Prostate cancer cells are sensitive to many types of chemotherapeutics, but most current protocols employ Taxol, prednisone and mitoxantrone [4]. Other agents evaluated have

${ }^{*}$ Corresponding author: Dr. Marvin Rubenstein, Chairman-Division of Cellula Biology, Hektoen Institute for Medical Research, 2240 West Ogden Avenue, 2'nd floor, Chicago, IL 60612, United States, Tel: +1 312864 4621; Fax: +1 312768 6010; E-mail: DrMarv@Prodigy.net

Received August 26, 2011; Accepted November 15, 2011; Published November 17,2011

Citation: Rubenstein M, Hollowell CMP, Guinan P (2011) Enhanced Delivery of Chemotherapeutic Alkylating Agents into Prostate Cancer Cells Employing the Androgen Receptor as Delivery Vehicle. Metabolomics 1:103. doi:10.4172/21530769.1000103

Copyright: (C) 2011 Rubenstein M, et al. This is an open-access article distributed under the terms of the Creative Commons Attribution License, which permits unrestricted use, distribution, and reproduction in any medium, provided the original author and source are credited. 
Citation: Rubenstein M, Hollowell CMP, Guinan P (2011) Enhanced Delivery of Chemotherapeutic Alkylating Agents into Prostate Cancer Cells Employing the Androgen Receptor as Delivery Vehicle. Metabolomics 1:103. doi:10.4172/2153-0769.1000103

included Cytoxan, DES, various platinates and even gene therapy. If innovatively delivered and suitably administered, gene therapy and alkylating agents (Cytoxan; cyclophosphamide) have potential advantages for specific tumor targeting and DNA inactivation.

Gene therapy: Gene function in prostate cancer is highly unstable. Many genes are altered in expression; some enhanced (like growth factors and their receptors), others are suppressed (PTEN). Therefore, gene therapy for prostate cancer has consisted of two types: 1) translational arrest, or 2) gene replacement. Translational arrest often employs the administration of antisense oligonucleotides (oligos), which has been demonstrated in both in vitro and in vivo models to suppress expression of many proteins [5] when directed against protein growth factors (EGF, TGF- $\alpha$, IGFBP), their receptors (EGFR) or inhibitors of apoptosis (bcl-2, clusterin). Gene replacement has concentrated on PTEN, a prostate cancer suppressor gene which negatively regulates the Akt/PKB signaling pathway [6].

Alkylating agent cytoxan: Cytoxan (Figure 1) is a low molecular weight (MW 261d) alkylating agent which adds an alkyl group $\left(\mathrm{C}_{\mathrm{n}} \mathrm{H}_{2 \mathrm{n}+1}\right)$ to guanine bases of DNA at the number 7 nitrogen atom of the imidazole ring. It's administered as a prodrug which is transformed by liver enzymes to an active phosphoramide mustard metabolite. It initiates cell death by forming intrastrand crosslinkages between opposing guanine residues.

Targeting strategy and delivery vehicle: Most chemotherapeutics for disseminated prostate cancer are administered intravenously. This approach subjects even normal cells to the cytotoxic effects of these agents. Selective toxicity is based on the premise that most tumors have proportionally more cells undergoing mitotic division with DNA synthesis than normal cells. To specifically direct agents more directly to tumors various techniques have been employed. Among these, monoclonal antibodies are the most specific and radiolabeled $\left({ }^{111} \mathrm{In}\right)$ anti-PSMA (CYT-356) has been used to image metastatic prostate tumors [7]. Our data has shown that in an in vivo model monoclonals directed against PSMA are more effective than those against secreted PSA when delivering antisense oligos. We have also shown that bispecific monoclonals (having active sites specific for biotin and either PSA or PSMA) can deliver biotinylated oligos specifically to prostate cells [8]. In a recent report a bivalent (antisense-like) PSMA binding aptamer-siRNA transcript was used to target eukaryotic elongation factor-2 mRNA [9].

Ideal agent: An ideal delivery agent would be one which specifically targets tumor cells, enhances entry into the cell and directs it to transcriptionally active sites on DNA. I propose coupling cyclophosphamide (Cytoxan) to the androgen analog R1881 (Figure 2) and using the AR itself as the delivery agent. Cytoxan would be transported into the nucleus and cross link DNA in those cells expressing this receptor.

R1881: R1881 is a synthetic agonist which binds to the androgen receptor with high affinity $\left(\mathrm{Kd}=1.7-1.9 \times 10^{-9} \mathrm{M}\right)$ [10]. Also known as methyltrienolone and, having a molecular weight of 284, when coupled to Cytoxan, it would still yield a relatively small conjugate for intracellular transport mediated via the AR. Although a potent agonist itself, R1881 when coupled to this alkylating agent the Cytoxan could be even more lethal if the cell has been androgen (agonist) activated.

The hypothesis: The hypothesis being proposed here is that a conjugate formed between R1881 and Cytoxan could specifically be recognized by prostate cancer cells. This bound conjugate would be taken up by these cells, transported to the nucleus and bind to transcriptionally active DNA sites, forming cross linkages between guanine residues on opposing strands. Rather than be stimulated by the androgen receptor, these cells would die due to their inability to replicate and separate their DNA. Specific uptake by prostate cancer cells would presumably minimize the risk of hepatotoxicity produced by R1881.

The Cytoxan/R1881 conjugate would be formed by either covalent linkage between the molecules with/or without a spacer segment; or both entities could be recognized by bivalent monoclonals having active sites against each.

Evaluation of the hypothesis: This hypothesis could be tested in vitro using the LNCaP tumor model which expresses the androgen receptor.

LNCaP cells would be seeded into replicate depressions of a 96 well plate. When approximately $75 \%$ confluent either Cytoxan alone or the Cytoxan/R1881 conjugate would be added to each well. An $\mathrm{LD}_{50}$ for Cytoxan would be used, which (in our experience) corresponds to approximately $40 \mathrm{~nm}$ for in vitro culture. Cells would be incubated for 24-48 hrs before solutions are aspirated and re-incubated for an additional $48 \mathrm{hrs}$ in $200 \mu \mathrm{l}$ of media. Cell counts can be determined following the addition of WST-1 reagent to each well, and after $2 \mathrm{hrs}$ the color intensity is measured by a microplate reader at a wavelength of $450 \mathrm{~nm}$, using a reference wavelength of $650 \mathrm{~nm}$.

Statistics: Microsoft Excel software can be utilized to calculate means and standard deviations. Student $t$ tests would determine significance.

\section{Discussion}

This year (2011) the American Cancer Society estimates that in spite of early detection, screening for prostate specific antigen (PSA)

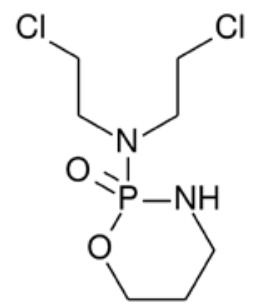

Image downloaded from Wikipedia and released into the public domain by author

Figure 1: Cyclophosphamide (Cytoxan).

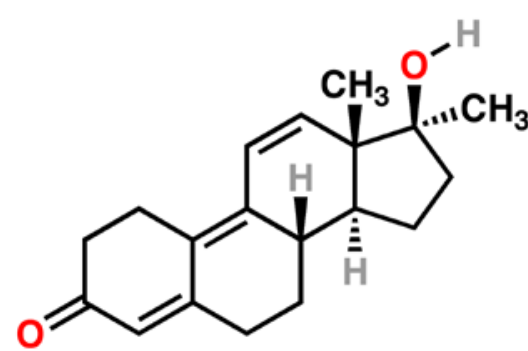

Image downloaded from Wikipedia and released into the public domain by author

Figure 2: Methyltrienolone (R1881). 
Citation: Rubenstein M, Hollowell CMP, Guinan P (2011) Enhanced Delivery of Chemotherapeutic Alkylating Agents into Prostate Cancer Cells Employing the Androgen Receptor as Delivery Vehicle. Metabolomics 1:103. doi:10.4172/2153-0769.1000103

Page 3 of 3

and effective treatments for localized disease, in the United States there will be 33,720 deaths from prostate cancer in addition to 240,890 newly diagnosed cases. However, even for patients choosing radical prostatectomy there remains a small percentage remaining at risk for recurrent disease. For many, the earliest indication of biochemical relapse is an increase in PSA levels and, at least for a while, many of these tumors continue to exhibit androgen sensitivity. For these patients chemotherapy targeting androgen receptor expressing cells could be an option. Unfortunately, as tumors progress, androgen receptor activity either diminishes or the receptor itself is subject to mutations which can prevent androgen binding or contribute to AR constituitive activity in androgen absence (even following hormone deprivation therapy). Therefore, the window of opportunity for this type of therapy may be limited. However, the current failure to develop effective chemotherapy for these patients makes any advance of potential interest. In tumors which are already androgen insensitive, other receptors can be used as delivery vehicles. Many are overexpressed in prostate tumors (like epidermal growth factor receptor; EGFR) which also internalizes various bound ligands (EGF and transforming growth factor- $\alpha$ ). Cytoxan could be similarly coupled to these ligands. Perhaps not as efficient as the androgen receptor as a delivery vehicle, any increase in specific cellular uptake is potentially useful.

\section{Acknowledgments}

The Cellular Biology laboratory at the Hektoen Institute is supported, in part, by the Blum Kovler Foundation, the Cancer Federation, Safeway/Dominicks Campaign for Breast Cancer Awareness, Lawn Manor Beth Jacob Hebrew Congregation, the Max Goldenberg Foundation, the Sternfeld Family Foundation, and the Herbert $\mathrm{C}$. Wenske Foundation.

\section{References}

1. Mooradian AD, Morley JE, Korenman SG (1987) Biological actions of androgens Endocr Rev 8: 1-28

2. Jia L, Coetzee GA (2005) Androgen receptor-dependent PSA expression in androgen-independent prostate cancer cells does not involve androgen receptor occupancy of the PSA locus. Cancer Res 65: 8003-8008.

3. Ghosh A, Wang X, Klein E, Heston WD (2005) Novel role of prostate-specific membrane antigen in suppressing prostate cancer invasiveness. Cancer Res 65: 727-731.

4. Petrylak DP, Tangen CM, Hussain MHA, Primo NL Jr, Jones JA, et al. (2004) Docetaxel and estramustine compared with mitoxantrone and prednisone for advanced refractory prostate cancer. N Engl J Med 351: 1513-1520.

5. Rubenstein M, Tsui $P$, Guinan $P$ (2004) A review of antisense oligo- nucleotides in the treatment of human disease. Drugs of the Future 29: 893-909.

6. Huang H, Cheville JC, Pan Y, Roche PC, Schmidt LJ, et al. (2001) PTEN induces chemosensitivity in PTEN-mutated prostate cancer cells by suppression of Bcl-2 expression. J Biol Chem 276: 38830-38836.

7. Feneley MR, Jan H, Granowska M, Mather SJ, Ellison D, et al. (2001) Imaging with prostate-specific membrane antigen (PSMA) in prostate cancer. Prostate Cancer and Prostatic Diseases 3: 47-52.

8. Mirochnik Y, Rubenstein M, Guinan P (2004) Two constructed antibody derived vehicles for targeting oligonucleotides to prostate tumors expressing prostate specific antigen. Drug Deliv 11: 161-167.

9. Wullner U, Neef I, Eller A, Kleines M, Tur MK, et al. (2008) Cell-specific induction of apoptosis by rationally designed bivalent aptamer-siRNA transcripts silencing eukaryotic elongation factor 2. Curr Cancer Drug Targets 8: 554-565.

10. Vittek J, Hernandez MR, Wenk EJ, Rappaport SC, Southern AL (1985) Binding of $3 \mathrm{H}$-methyltrienolone $\left({ }^{3} \mathrm{H}-\mathrm{R} 1881\right)$ to androgen receptors in human gingival. $J$ Peridontal Res 20: 41-46. 